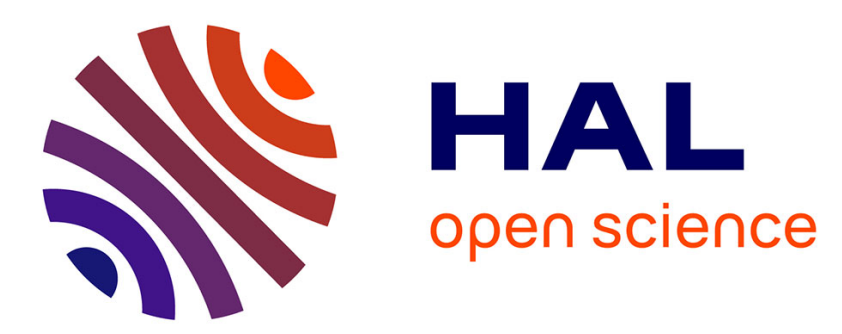

\title{
Hand preferences in human adults: Non-communicative actions versus communicative gestures
}

Hélène Cochet, Jacques Vauclair

\section{To cite this version:}

Hélène Cochet, Jacques Vauclair. Hand preferences in human adults: Non-communicative actions versus communicative gestures. Cortex, 2012, 48 (8), pp.1017-1026. 10.1016/j.cortex.2011.03.016 . halshs-01464512

\section{HAL Id: halshs-01464512 \\ https://shs.hal.science/halshs-01464512}

Submitted on 11 Jul 2017

HAL is a multi-disciplinary open access archive for the deposit and dissemination of scientific research documents, whether they are published or not. The documents may come from teaching and research institutions in France or abroad, or from public or private research centers.
L'archive ouverte pluridisciplinaire HAL, est destinée au dépôt et à la diffusion de documents scientifiques de niveau recherche, publiés ou non, émanant des établissements d'enseignement et de recherche français ou étrangers, des laboratoires publics ou privés. 
Provided for non-commercial research and education use. Not for reproduction, distribution or commercial use.

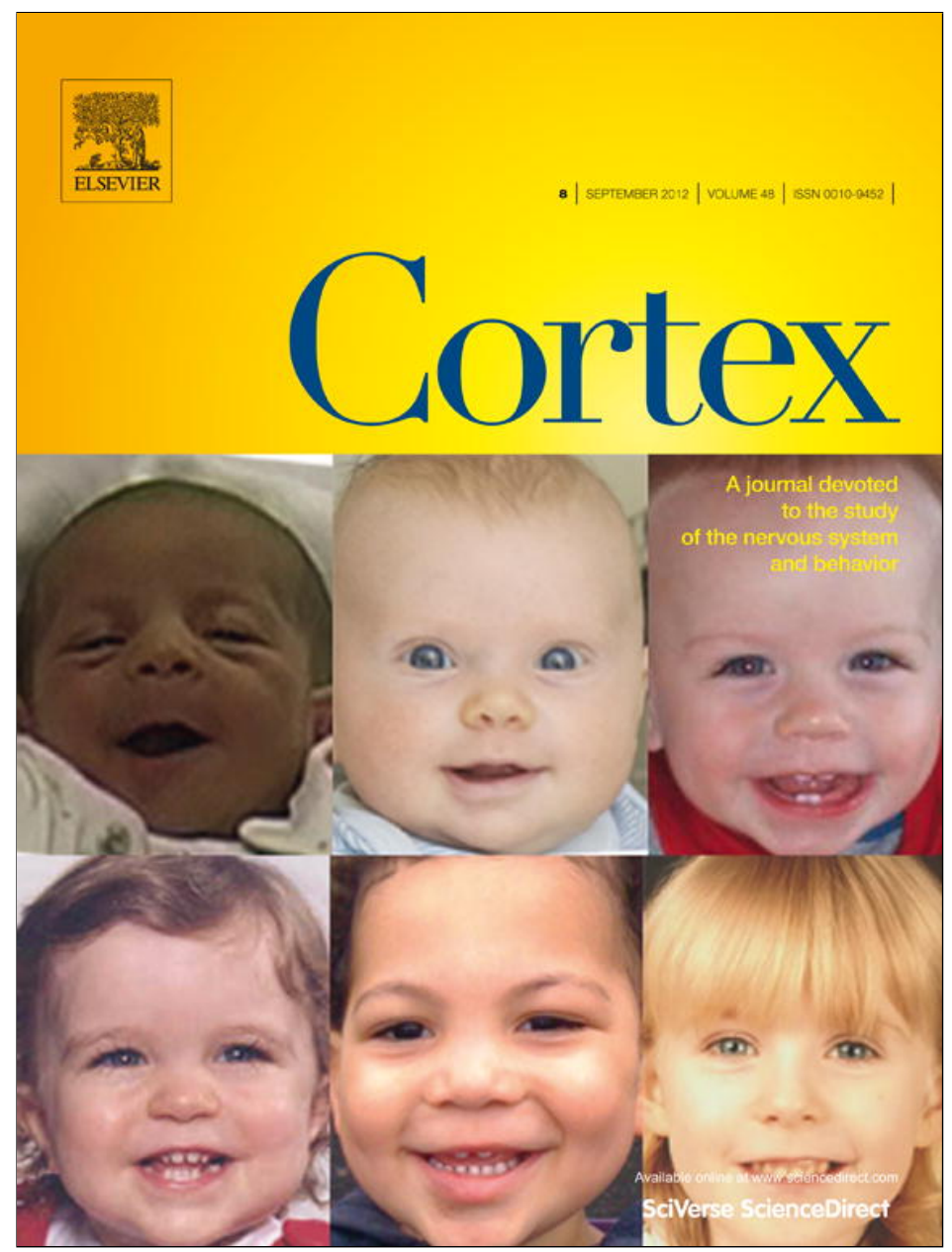

This article appeared in a journal published by Elsevier. The attached copy is furnished to the author for internal non-commercial research and education use, including for instruction at the authors institution and sharing with colleagues.

Other uses, including reproduction and distribution, or selling or licensing copies, or posting to personal, institutional or third party websites are prohibited.

In most cases authors are permitted to post their version of the article (e.g. in Word or Tex form) to their personal website or institutional repository. Authors requiring further information regarding Elsevier's archiving and manuscript policies are encouraged to visit:

http://www.elsevier.com/copyright 


\title{
Research report
}

\section{Hand preferences in human adults: Non-communicative actions versus communicative gestures}

\author{
Hélène Cochet and Jacques Vauclair* \\ Research Centre in the Psychology of Cognition, Language \& Emotion, Aix-Marseille University, Department of Psychology, \\ Aix-en-Provence, France
}

\section{A R T I C L E I N F O}

\section{Article history:}

Received 5 November 2010

Revised 22 February 2011

Accepted 28 March 2011

Action editor Alan Beaton

Published online 7 April 2011

\section{Keywords:}

Handedness

Bimanual manipulation

Pointing gestures

Speech

Lateralization

\begin{abstract}
A B S T R A C T
Hand preferences for pointing gestures and bimanual manipulative activities were investigated in 127 adult participants. Pointing gestures were produced in two different conditions: a speech condition, in which the gestures were accompanied by speech, and a silent condition. Although the classification of participants as left- or right-handers, or ambidextrous, was consistent across the manipulation and pointing tasks for $85 \%$ of participants, results showed only moderate correlations between handedness scores for bimanual manipulation and pointing gestures. Moreover, results did not reveal any difference in the degree of hand preference between pointing gestures produced along with speech and gestures produced on their own. The implications of these findings are discussed in relation to the lateralization of non-communicative manual actions, communicative gestures and speech.
\end{abstract}

(ㄷ) 2011 Elsevier Srl. All rights reserved.

\section{Introduction}

The concept of "handedness" has traditionally referred to manipulative actions, and only a few researchers have investigated manual specialisation for communicative behaviour. Furthermore, although some studies have already undertaken comparisons between manual preferences for manipulation and communication in non-human primates (in chimpanzees: Hopkins et al., 2005; in baboons: Meguerditchian and Vauclair, 2009), as well as in human infants (Bates et al., 1986; Cochet and Vauclair, 2010a; Vauclair and Imbault, 2009), this issue has never been directly addressed in human adults. In an attempt to investigate the relationship between hand preference and lateralization of speech processing, the main purpose of the present study was thus to compare handpreference patterns for non-communicative actions and communicative gestures in human adults.

\subsection{Relationship between hand preference and speech processing}

Speech being one of the most striking lateralized functions of the human brain, its relationship with handedness has long been of interest to researchers. In order to investigate this relationship, the nature of the asymmetric actions being performed needs to be taken into account, leading to the

\footnotetext{
* Corresponding author. Research Centre in the Psychology of Cognition, Language \& Emotion, Aix-Marseille University, Department of Psychology, 29, av. Robert Schuman, 13621 Aix-en-Provence Cedex 1, France.

E-mail addresses: helene.cochet@univ-provence.fr (H. Cochet), Jacques.Vauclair@univ-provence.fr (J. Vauclair).

URL: http://sites.univ-provence.fr/wpsycle/index.html 0010-9452/\$ - see front matter @ 2011 Elsevier Srl. All rights reserved. http://dx.doi.org/10.1016/j.cortex.2011.03.016
} 
distinction between purely manipulative activities and activities involving a communicative intention, that is, communicative gestures. Concerning manipulative actions, the left cerebral hemisphere was shown to be dominant for language in $96 \%$ of right-handers and $70 \%$ of left-handers (Knecht et al., 2000). This means that the vast majority of left-handed individuals do not have right-hemisphere dominance for speech, demonstrating that the relationship between handedness for manipulative activities and language lateralization is very indirect. For this reason, studying the asymmetries that pertain to communicative behaviour may bring a new perspective to the relationship between hand preference and language processing.

First, behavioural studies have reported a right-sided bias for gestures that spontaneously accompany speech in adults (e.g., Dalby et al., 1980; Kimura, 1973; Saucier and Elias, 2001). Some of these gestures are used intentionally, to refer directly to the speech content (e.g., iconic gestures), but most hand movements are produced to lend continuity, emphasis and rhythm to speech, or else are not clearly connected with its discursive structure (e.g., self-touching movements). Moreover, it has been argued that co-speech gestures are independent of speech production processes (Chu and Kita, 2009). Therefore, in order to examine speech-gesture links and to compare hand-preference patterns for non-communicative actions and communicative gestures, it seems more appropriate to focus on gestures that have the clearest communicative intention. In infants and children, a right-sided asymmetry has been observed for communicative gestures such as pointing gestures and/or symbolic gestures (e.g., Bates et al., 1986; Blake et al., 1994; Cochet and Vauclair, 2010a, 2010b; Vauclair and Imbault, 2009; Young et al., 1985). In deaf adults, a right-sided bias has been reported for signing (e.g., Grossi et al., 1996; Vaid et al., 1989), which may be viewed in relation to neuroimaging data showing that Broca's area is activated in the production of sign language (e.g., Corina et al., 2003; Emmorey et al., 2007). In hearing adults, one study has reported a right-sided asymmetry for pointing gestures (Bryden et al., 2000), however, the pointing task used in that study did not involve any communicative intention (participants were asked to point to an object indicated by the experimenter, without any specific communicative motive). So for now, no data are available for intentional communicative gestures produced by hearing adults.

Second, studies using event-related potentials and functional imaging have shown that Broca's area is involved in the interaction between words and gestures (e.g., Özyürek et al., 2007; Xu et al., 2009). Moreover, changes in arm kinematics and voice parameters have been reported when symbolic gestures and the corresponding words are simultaneously produced, compared with conditions under which words or gestures are performed on their own (Barbieri et al., 2009; Bernardis and Gentilucci, 2006). Furthermore, neuropsychological studies have revealed that the link between aphasia and apraxia in adults is mainly restricted to ideomotor apraxia, that is, to the reproduction of symbolic and meaningful gestures (see Bates and Dick, 2002).

Altogether, these findings emphasized a tight interconnection in the brain between speech and gesture; nevertheless, neurophysiological and behavioural evidence have suggested that the control of manual actions (which includes both communicative gestures and non-communicative activities) and language processing involves complex cerebral networks (e.g., Gentilucci and Dalla Volta, 2008; Iverson and Thelen, 1999). For example, a functional magnetic resonance imaging (fMRI) study recently reported that the observation of a human right hand grasping an object and the observation of that hand pointing towards the same object result in similar activation of the premotor cortex, an area that plays an important role in coding the observation of manual action (Pierno et al., 2009). By contrast, results of this latter study also suggested different relationships between grasping versus pointing and the cerebral control of speech. Indeed, the comparison of the grasping and control conditions (in the control condition, participants observed a palm-down hand resting next to the object) revealed bilateral differential activity, whereas the differential activation between the pointing and control conditions was confined to the left cerebral hemisphere. Moreover, Gonzalez and Goodale (2009) showed that the more participants used their right hand for precision grasping, the more language was lateralized to the left hemisphere, but the rather low correlation value indicated that more complex processes may come into play.

Overall, these results indicate that the cerebral control of communicative behaviours may not be entirely independent of the system involved in purely manipulative activities. The comparison of hand preferences for communicative gestures and object manipulation may therefore improve our understanding of these interactions.

\subsection{Comparison between manipulative activities and communicative gestures}

In young children, the right-sided asymmetry appears to be stronger for pointing gestures than for manipulative actions (Bates et al., 1986; Vauclair and Imbault, 2009). Signed gestures produced by children born to deaf parents have also been reported to be more right-handed than other manual activities (Bonvillian et al., 1997). A stronger right-handed bias for communicative gestures has been observed in non-human primates as well (in chimpanzees: Hopkins et al., 2005; in baboons: Meguerditchian and Vauclair, 2009). Moreover, correlational analyses in non-human primates and human children have revealed that hand preference for communicative gestures does not significantly correlate with handedness scores for manipulative actions (e.g., Cochet and Vauclair, 2010a), although a weak correlation between these two measures has been reported in toddlers (Vauclair and Imbault, 2009). These different patterns of laterality highlight the absence of a strong relationship between communicative gestures and manipulative actions, which has led researchers to hypothesise that a specific communication system in the left cerebral hemisphere, distinct from the system involved in non-communicative motor activities, may control both gestural and vocal communication, at least in human infants and non-human primates.

In human adults, Bryden et al. (2000) examined hand preferences for different unimanual actions, including grasping and pointing towards small objects in different regions of hemispace. The authors failed to observe any difference in the frequency of right hand use across the different tasks, but did 
not investigate correlations between the tasks. Therefore, the relationship between handedness for manipulative actions and communicative gestures needs to be examined further in adults. From this perspective, it is important to consider the methodological issues related to the study of handedness for manipulative activities, which, as outlined hereafter, may complicate comparisons across studies.

\subsection{Handedness for manipulation}

Even though researchers seem to agree on a mean percentage of $90 \%$ of right-handers in adults (Annett, 1985; Medland et al., 2004; Raymond and Pontier, 2004), the study of handedness for manipulation raises several problems, not least those related to the definition of handedness itself. Handedness is generally defined as the preferred use of one hand for a specific task, regardless of performance, but it can also refer to the hand that is faster and more precise for that task (e.g., Healey et al., 1986). Even if most of the time, people preferentially use their more dexterous hand for a given task, these two definitions may not always perfectly coincide. For example, some people are equally skilled with both hands, but still prefer using one hand rather than the other (see Kraus, 2005). In addition, handedness can be assessed either through self-reported questionnaires, which can be regarded as somewhat subjective, in that they require participants to imagine or recall which hand they use or would use for a given activity, or through direct observation of manual activity.

Moreover, some researchers focus on unimanual manipulations, while others study the coordination of the two hands in bimanual activities, both hands having differentiated roles. This distinction is particularly important, as task complexity has been shown to influence the degree of handedness in both adults and children (Fagard and Lockman, 2005; Fagard and Marks, 2000; Flowers, 1975; Provins and Glencross, 1968), as well as in non-human primates (Fagot and Vauclair, 1991). Thus, bimanual manipulative actions seem to induce more lateralized patterns than less challenging tasks, such as simple object grasping.

The final issue concerns the relevance of classifying individuals as left- or right-handers, given that hand preferences are continuously distributed across a spectrum from strongly left-handed to strongly right-handed, and that this categorization relies on different criteria across studies (e.g., Beaton, 2003; Hopkins, 1999).

\subsection{Comparison of gesture laterality in speech and silent conditions}

Finally, another way of investigating speech-gesture links is to compare the degree of asymmetry between gestures produced simultaneously with speech and gestures produced on their own. Kimura (1973) observed an increase in the frequency of spontaneous right-handed movements during speaking, compared with silent conditions, whereas the occurrence of left-handed movements was not affected. Thus, hand preference was stronger when gestures were accompanied by speech, once again suggesting an association between the control of speech and that of gestures in the left cerebral hemisphere. However, the gestures examined in this study were free movements, defined as "any motion of the limb which did not result in touching of the body or coming to rest" (p. 46), such as waves of the hand. This broad definition may cast doubt upon the intentional and communicative nature of these movements, and we therefore have to ask whether the activation of the speech system affects the laterality of proven intentional gestures (e.g., pointing gestures) in a similar way.

In a bid to answer this question, Lausberg and Kita (2003) asked adult participants to use hand gestures to describe the content of animations showing different movements of geometric objects, either with or without speech. These authors did not report any difference between the silent and speech conditions in the degree of hand preference for iconic gestures. They did, however, observe different distributions of unimanual and bimanual gestures, with unimanual gestures being more frequently produced in the speech condition and bimanual gestures in the silent one. The authors did not distinguish between the activities of the two hands for bimanual gestures, even though they may have had different roles, to the extent that one hand could have been regarded as dominant. The comparison of hand-preference patterns for these two conditions thus does not allow us to draw any further conclusions. In toddlers, Cochet and Vauclair (2010b) found that pointing gestures accompanied by vocalizations were no more right-handed than gestures produced on their own. By contrast, in chimpanzees, Hopkins and Cantero (2003) observed a greater degree of right-handedness when food-begging gestures were accompanied by vocalizations, compared with the same gestures produced on their own.

Accordingly, evidence still need to be collected in human adults to determine whether the left cerebral hemisphere is more highly activated when speech and communicative gestures intentional and referential - are produced simultaneously.

In the present study, we therefore sought to examine the relationship between hand preference and lateralization of speech processing. We compared the degree of hand preference (1) between pointing gestures and bimanual manipulative activities and (2) between pointing gestures produced on their own and gestures produced along with speech. First, we expected any correlations we found between the degrees of hand preference for pointing gestures and for coordinated bimanual manipulations to be only weak to moderate. Second, we hypothesised that the right bias of pointing gestures accompanied by speech would be stronger than the bias of gestures produced on their own.

\section{Method}

\subsection{Participants}

The participants were 127 French university students between 18 and 48 years of age $[M=21.9$ years, standard deviation $(\mathrm{SD})=5.8$ years], including 56 men and 71 women .

\section{2. $\quad$ Procedure and materials}

Participants were tested individually in a university experimental room. They were seated at a rectangular table, with 
the experimenter sitting opposite them. The experiment included a pointing task and a manipulation task, and lasted for a total of approximately $30 \mathrm{~min}$. In order to control their posture, participants were asked to place their hands on the table between each trial, on two symmetrical stickers that had been positioned $25 \mathrm{~cm}$ away from the edge of the table. Participants were told that we wanted to study the perception and judgement of different photographs and thus did not know that we were recording hand preferences. They were all informed by e-mail of the real purpose of the study once the data collection was over (we did not inform the participants immediately after the experiment to prevent them from communicating the information to their fellow participants).

\subsubsection{Communicative gestures: pointing task}

In order to elicit pointing gestures, the experimenter showed participants several photographs and asked them to point to the one they preferred. In order to be sure that the photographs were free of any emotional content that might influence patterns of laterality (e.g., Bourne, 2008; Bryden et al., 1991; Everhart et al., 1996), we selected images of neutral valence from the International Affective Picture System (IAPS; Lang et al., 1999). These IAPS photographs were divided into 30 sets of four photographs. Each set of four photographs (each measuring approximately $8 \times 5 \mathrm{~cm}$ ) was printed on an A4 sheet in a single column. The experimenter placed the sheets one at a time on the table, approximately $.6 \mathrm{~m}$ away from the participants, so that they had to extend their arms when pointing and could not touch the photographs.

In order to compare the degree of the right-sided bias when both gestural and vocal modalities were involved and when gestures were produced on their own, the trials were administered in two conditions, whose order of presentation was alternated across participants. In the silent condition, participants were asked to indicate their favourite photograph through gestures, without saying a word. The experimenter stressed this requirement and reiterated it during the session, when necessary. In the speech condition, participants had to express their choice simultaneously gesturally and verbally, and briefly justify their choice as they pointed. Participants were told that these two conditions were set up in order to study the influence of speech on perception. There were 15 trials in each condition.

\subsubsection{Manipulative action: bimanual coordination task}

Handedness for non-communicative actions was assessed by means of a bimanual coordination task. The experimenter placed a cylinder-shaped container filled with several pieces of paper on the table, in front of the participants. This container was approximately $25 \mathrm{~cm}$ tall, meaning that participants had to tilt it with one hand while the other hand grabbed one of the pieces of paper. A number was written on each of these pieces and participants were told that they had to take one to determine the order of image presentation for the second task (pointing task). For example, if a participant picked out a paper on which a three was written, the experimenter showed him or her the third set of photographs. Another number then had to be picked out, and so on, until 30 trials had been performed in each task. Trials for the pointing and manipulation tasks were thus alternated. This procedure allowed us to randomise the sets of photographs and also provided us with a plausible motive for the manipulation task.

\subsubsection{Manipulative activities}

At the beginning of the experiment, participants filled in a questionnaire about their name, age and e-mail address, which allowed the experimenter to record the hand used for writing. Additional measures of hand preference for manipulative activities were collected through a hand-preference questionnaire, which was sent by e-mail to the participants at the end of all the experiments. Eighty-three participants (i.e., $65.4 \%$ of the sample) answered the questionnaire. This questionnaire contained 13 items extracted from the Edinburgh Handedness Inventory (Oldfield, 1971), including one item about handwriting. The latter confirmed the results obtained from direct observation during the experiment, for all the participants who answered the questionnaire.

\subsection{Data analyses}

An individual handedness index score (HI) was calculated for each participant and for the different tasks using the formula $(\mathrm{R}-\mathrm{L}) /(\mathrm{R}+\mathrm{L})$, where $\mathrm{R}$ and $\mathrm{L}$ stood for the total right- and lefthand responses. The HI values lay along a continuum from -1 to 1 , with the sign indicating the direction of hand preference and the absolute value (AbsHI) characterising the strength of hand preference. For the bimanual task, the hand that played an active role, that is, that grabbed the piece of paper, was considered as the dominant hand and the one having a supporting role, that is, tilting the container, as the nondominant hand. This distinction between active and passive roles for the two hands has been widely used in studies with human infants or non-human primates, for example with the tube task, in which the non-dominant hand grasps a tube while the dominant hand picks up the object or food inserted in it (e.g., Hopkins et al., 2005; Vauclair and Imbault, 2009).

Moreover, binomial tests performed for each individual, indicating whether the use of the left and right hands significantly differed, enabled us to classify participants as left-handed, right-handed or ambidextrous in each task. Given that all participants performed the same number of trials, we calculated the number of left- and right-hand responses allowing this classification, the level of significance being set at .05. For the manipulation task, individuals were considered left- or right-handed if they performed at least 20 of the 30 trials with the left or the right hand (respectively), and as ambidextrous if the number of righthand responses varied between 11 and 19. For the handedness questionnaire, participants were considered left- or right-handed if they answered that they used their left or right hand (respectively) for at least 10 of the 13 items, and as ambidextrous if the number of right-hand responses varied between 4 and 9. Last, for the pointing tasks, participants were classified as left- or right-handers if they performed at least 11 of the 15 trials with the left or the right hand (respectively), and as ambidextrous if the number of righthand responses varied between 5 and 10. 


\begin{tabular}{|c|c|c|c|c|}
\hline & $\begin{array}{l}\text { Bimanual } \\
\text { manipulation } \\
\text { (30 trials) }\end{array}$ & $\begin{array}{l}\text { Handedness } \\
\text { questionnaire } \\
\text { (13 items) }\end{array}$ & $\begin{array}{c}\text { Pointing } \\
\text { (silent condition) } \\
\text { (15 trials) }\end{array}$ & $\begin{array}{c}\text { Pointing } \\
\text { (speech condition) } \\
\text { (15 trials) }\end{array}$ \\
\hline Mean number of right-hand responses & $26.39( \pm 8.85)$ & $10.73( \pm 3.68)$ & $12.59( \pm 4.83)$ & $12.10( \pm 4.70)$ \\
\hline Mean HI & $.76( \pm .59)$ & $.73( \pm .52)$ & $.68( \pm .64)$ & $.65( \pm .62)$ \\
\hline Mean AbsHI & $.94( \pm .18)$ & $.88( \pm .17)$ & $.91( \pm .22)$ & $.85( \pm .28)$ \\
\hline
\end{tabular}

\section{Results}

\subsection{Hand preference: descriptive results}

\subsubsection{Bimanual manipulation}

For the coordinated bimanual task, 111 participants were right-handed (87.4\%), 12 were left-handed $(9.4 \%)$ and four were ambidextrous (3.2\%). Mean HI was $.76(\mathrm{SD}=.59)$ and mean AbsHI was .94 $(\mathrm{SD}=.18)$.

\subsubsection{Handedness questionnaire}

Of the 83 participants who answered the questionnaire, 71 were right-handed (85.54\%), seven left-handed (8.44\%) and five ambidextrous (6.02\%). Mean HI was $.73(\mathrm{SD}=.52)$ and mean AbsHI was $.88(\mathrm{SD}=.17)$. Handedness scores measured with this questionnaire were not correlated with the $\mathrm{HI}$ obtained from the coordinated bimanual task $(r=.11$; ns). However, when we distinguished between right-handed, lefthanded and ambidextrous participants (on the basis of handedness scores on the bimanual manipulation task), a significant correlation was observed between these two measures in right-handed individuals $(r=.39 ; p<.001)$. There was no significant correlation in left-handed participants $(r=.33 ; n s)$, and there were too few ambidextrous individuals for us to perform the correlation.

\subsubsection{Pointing gestures}

For the pointing task in the silent condition, 103 participants were right-handed (81.1\%), 16 were left-handed (12.6\%) and eight were ambidextrous (6.3\%). Mean HI was .68 (SD = .64) and mean AbsHI was .91 ( $\mathrm{SD}=.22)$. For the pointing task in the speech condition, 98 participants were right-handed (77.2\%), 13 were left-handed (10.2\%) and 16 were ambidextrous (12.6\%). Mean HI was .65 (SD =.62) and mean AbsHI was . $85(\mathrm{SD}=.28)$.

\begin{tabular}{|c|c|c|c|c|}
\hline & \multicolumn{4}{|c|}{ Manipulation } \\
\hline & $\begin{array}{l}\text { Right- } \\
\text { handed }\end{array}$ & $\begin{array}{c}\text { Left- } \\
\text { handed }\end{array}$ & Ambidextrous & Total \\
\hline \multicolumn{5}{|l|}{ Pointing } \\
\hline Right-handed & 98 & 2 & 3 & 103 \\
\hline Left-handed & 7 & 9 & 0 & 16 \\
\hline Ambidextrous & 6 & 1 & 1 & 8 \\
\hline Total & 111 & 12 & 4 & 127 \\
\hline
\end{tabular}

These descriptive results, as well as the mean number of right-hand responses for each activity, are summarized in Table 1.

\subsection{Comparison of hand-preference patterns for bimanual manipulation and pointing gestures}

There was no significant difference between pointing gestures produced on their own, that is, in the silent condition, and bimanual manipulative actions, either for mean $\mathrm{HI}$, $t(127)=1.57$; $n s$, or for mean AbsHI, characterising handpreference strength, $t(127)=1.68$; ns. HIs for these two measures were significantly correlated $(r=.56 ; p<.001$ for $\mathrm{HI}$ and $r=.36 ; p<.001$ for AbsHI). By contrast, pointing gestures produced with speech were less right-handed than bimanual manipulative actions, $t(127)=2.55 ; p<.05$. The strength of handedness was also greater for manipulative actions, $\mathrm{t}(127)=4.46 ; p<.001$. HIs for these two measures were significantly correlated $(r=.65 ; p<.001$ for $\mathrm{HI}$ and $r=.55$; $p<.001$ for AbsHI). Comparison of these correlations using Steiger's (1980) t-test revealed that HI for bimanual manipulation was more strongly correlated with $\mathrm{HI}$ for pointing in the speech condition than with $\mathrm{HI}$ for pointing in the silent condition, $t=2.65 ; p<.01$.

Categorical analyses were also performed, based on the number of individuals classified as right-handed, left-handed and ambidextrous regarding bimanual manipulative actions and pointing gestures in the silent condition ${ }^{1}$ (see Table 2). Handedness patterns were consistent across the two different tasks for $85 \%$ of the participants, including $77.1 \%$ who were right-handed for both the pointing task and the coordinated bimanual task, $7.1 \%$ who were left-handed and .8\% who were ambidextrous.

However, the distinction between right- and left-handers regarding the manipulation task actually revealed an absence of any significant correlation between hand preferences for pointing and manipulation (in right-handers, $r=.059 ; n s$ and in left-handers, $r=.26 ; n s)$. The distinction between right- and left-handers regarding the pointing task led to similar results (in right-handers, $r=.13$; $n s$ and in lefthanders, $r=.23$; $n s)$, indicating that the significant correlation between pointing gestures and bimanual manipulation described earlier should be interpreted with caution.

\footnotetext{
${ }^{1}$ It seemed more relevant to focus on the condition involving the gestural modality alone, rather than on the bimodal condition, to differentiate between right-handers, left-handers and ambidextrous individuals.
} 


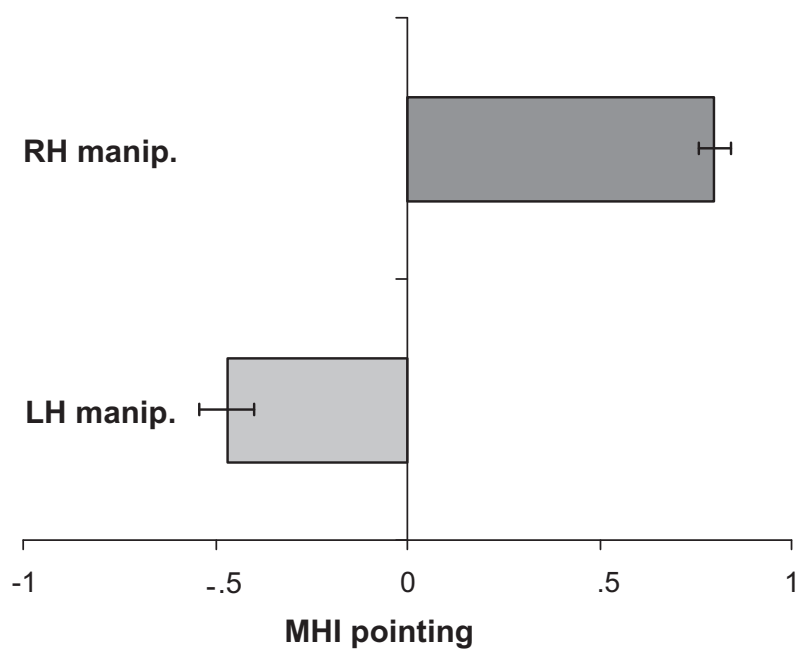

Fig. 1 - Mean HI ( \pm standard error) for pointing gestures in right- (RH) and left-handers (LH) for bimanual manipulation.

Moreover, as shown in Figs. 1 and 2, mean HI for pointing was $.80(\mathrm{SD}=.49)$ in right-handers for manipulation and -.47 $(\mathrm{SD}=.79)$ in left-handers for manipulation. Conversely, mean $\mathrm{HI}$ for bimanual manipulation was $.91(\mathrm{SD}=.34)$ in righthanders for pointing and $-.11(S D=100)$ in left-handers for pointing. The number of ambidextrous participants was too small for us to perform these analyses.

\subsection{Comparison of hand preferences for pointing in the silent and speech conditions}

Overall, pointing gestures accompanied with speech were not more right-handed than pointing gestures produced on their own, $\mathrm{t}(127)=1.23$; $n s$. HIs for these two variables were significantly correlated $(r=.88 ; p<.001)$. By contrast, when we focused on the strength of hand preference (i.e., AbsHI), the latter appeared to be greater for gestures produced on their

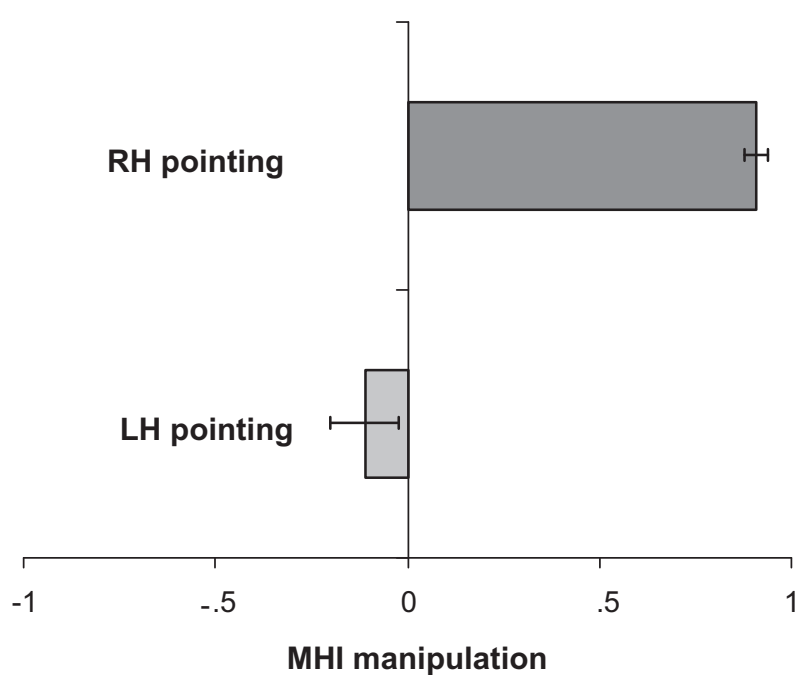

Fig. 2 - Mean HI ( \pm standard error) for bimanual manipulation in right- $(\mathrm{RH})$ and left-handers $(\mathrm{LH})$ for pointing gestures. own than for gestures produced along with speech, $t(127)=$ 2.64; $p<.01$. Pearson's correlation was also significant for AbsHI $(r=.50 ; p<.001)$.

Moreover, the correlations between HIs for gestures produced along with speech and gestures produced on their own were significant in right-handers (with respect to handpreference scores for pointing in the silent condition, $r=.54$; $p<.001)$, but not in left-handers $(r=-.07 ; n s)$ or ambidextrous individuals $(r=.23 ; n s)$.

Finally, the results did not reveal any effect of gender on handedness scores, regardless of whether they were associated with bimanual manipulation, $F(1,125)=.12$; $n$ s, or with pointing gestures in either the silent condition, $F(1,125)=3.70$; $n s$, or the speech one, $F(1,125)=1.49$; $n$.

\section{Discussion}

In the present study, handedness was assessed in a large sample of adults, through direct observation of hand use and also via a questionnaire. The main objective was to compare the degrees of hand preference for non-communicative actions (bimanual manipulation) and communicative gestures (pointing).

More than $87 \%$ of participants were right-handed for manipulation, in line with several studies reporting approximately $90 \%$ of right-handers among human adults (Annett, 1985; Raymond and Pontier, 2004). A strong majority of participants were also classified as right-handers for pointing gestures (approximately $81 \%$ in the silent condition and $77 \%$ in the speech condition). There was no significant difference in the mean HIs between pointing gestures produced on their own and bimanual manipulative actions, whereas pointing gestures produced along with speech were found to be less right-handed than bimanual manipulative actions. Thus, contrary to our initial hypothesis, lateralization of communicative gestures was no more robust than lateralization of noncommunicative motor actions, and was actually weaker when gestures were accompanied by speech. Moreover, overall results revealed significant, but moderate, correlations between handedness scores for pointing and for manipulation (the percentage of variance explained varied between $31 \%$ and $42 \%$, depending on the condition). However, further analyses that distinguished between right- and left-handers failed to reveal any significant correlations between hand preferences for pointing and manipulation.

Until now, the relationship between handedness patterns for gestures and non-communicative actions had not been directly investigated in human adults. As a consequence, no parallels can be drawn with other studies. Nevertheless, it may be useful to compare these findings with results of studies conducted in non-human primates and in human infants, at least with those that have used similar tasks and similar indexes to assess handedness. Mean HIs observed in the present study for manipulative activities were much stronger than the mean HIs that have been reported for nonhuman primates (in chimpanzees: Hopkins et al., 2005; in baboons: Meguerditchian and Vauclair, 2009) and for human children (e.g., Cochet and Vauclair, 2010a). For instance, measures of handedness in toddlers between 10 and 40 
months of age have revealed a mean HI of .32 (Vauclair and Imbault, 2009), while the mean HI observed in the present study reached .76. The degree of hand preference for manipulative actions therefore continues to increase strongly throughout the course of development, and not solely in infants and toddlers, as previously indicated by a study in children between 3 and 9 years of age (McManus et al., 1988).

Regarding communicative gestures, the degree of hand preference observed in the participants of the present study was also stronger than in non-human primates (Hopkins et al., 2005; Meguerditchian and Vauclair, 2009), although this comparison needs to be viewed with some caution, as the continuity between communicative gestures produced by nonhuman primates (e.g., food beg and hand slap gestures) and humans is still subject to debate (e.g., Gómez, 2005; Pika, 2008).

Moreover, hand preference for pointing gestures does not appear to differ strongly between children and adults (e.g., Vauclair and Imbault, 2009). The mean HIs reported for spontaneous pointing gestures (.68; Cochet and Vauclair, $2010 b)$ and informative pointing in toddlers (.70, Cochet and Vauclair, 2010a) are similar to the mean HIs observed in the present study (.68 and .65 in the silent and speech conditions, respectively). Therefore, under certain circumstances, adults and children aged approximately 1-3 years present an equivalent degree of left-hemisphere dominance in the production of communicative gestures, and overall, the difference in the distribution of hand-preference patterns between adults and infants is greater for object manipulation than for pointing gestures. These results indicate that hand preference for pointing gestures is established earlier in development than handedness for manipulative actions, thus suggesting that object manipulation is an unlikely basis for the emergence of right-handedness in humans. Moreover, the strong right-sided asymmetry reported for informative pointing in young children - an asymmetry comparable to the one observed in adults in the present study - suggests that cooperative abilities may play an important part in the development of a left-lateralized system of communication (Cochet and Vauclair, 2010a).

As previously stated, our results revealed moderate correlations between measures of handedness for pointing and manipulation, but these correlations proved not to be significant when right- and left-handers were considered separately. At a more general level, the categorization of participants as right- or left-handers for bimanual manipulation was not entirely independent of the categorization for pointing gestures. Here again, the contrast with non-human primates and human infants may help us to interpret these results and improve our understanding of issues related to the origins of handedness and human language. In non-human primates, researchers have shown that hand preferences for gestures are not significantly correlated with hand use for manipulative actions, whether these actions concern unimanual reaching or bimanual manipulation (in chimpanzees: Meguerditchian et al., 2010; in baboons: Meguerditchian and Vauclair, 2009), whereas handedness scores for different communicative gestures are significantly correlated with each other (hand slap and food beg gestures). In children, studies have also failed to reveal any significant correlation between hand preferences for pointing gestures and handedness scores for manipulative actions (e.g., Cochet and Vauclair, 2010a; Cochet et al., in press), although one study did report a weak correlation between these two measures, explaining $15 \%$ of the variance (Vauclair and Imbault, 2009).

Hand preferences for communicative gestures and for noncommunicative activities are thus quite independent in human infants and in non-human primates, whereas these two variables seem, to some extent, to be related in adults (although hand choices for these different activities do not perfectly coincide). This interconnection is supported by the results of an fMRI study, showing that the observation and production of communicative gestures and object-directed movements activate the mirror neuron system to a similar degree (Montgomery et al., 2007).

To summarize, hand preference for communicative gestures appears to be established in early development, whereas the increase in the degree of handedness for object manipulation seems to occur later in childhood. Language lateralization may thus initially be associated with the asymmetry of communicative gestures, with the gradual development of interactions between the cerebral control of speech, gestures and manipulative activities resulting in complex intertwined networks in human adults.

The second objective of the present study was to find out whether pointing gestures produced along with speech were more right-handed than gestures produced on their own. Results did not confirm our hypothesis, failing to reveal any overall difference in the degree of right-sided asymmetry for pointing gestures between the speech and silent conditions. We had expected pointing gestures accompanied by speech to be more right-handed than gestures produced on their own, given that the control of speech and gesture in the left cerebral hemisphere is mediated by very close, and possibly similar, neurobiological substrates (e.g., Bernardis and Gentilucci, 2006; Gentilucci and Dalla Volta, 2008; Xu et al., 2009). At first glance, one might thus interpret these findings as reflecting bilateral control of speech in our participants, and with hindsight, it would have been helpful to directly measure cerebral lateralization for speech, for example with a dichotic listening task. However, this bilateral hypothesis appears unlikely, as it is well acknowledged that the majority of people, even left-handers, have left-hemisphere dominance for language processing (e.g., Knecht et al., 2000), and the relatively large sample of the present study enables us to rule out the possibility of any sampling bias.

The fact that the "strengthening" effect of vocalizations on hand choice for communicative gestures has been demonstrated in chimpanzees (Hopkins and Cantero, 2003) offers several possible explanations for the results of the present study. First, the difference between ape vocalizations and human speech suggests that we should focus on the potential effect of discourse content on handedness patterns. It has been argued that the nature of the task, and more specifically the involvement of verbal versus spatial abilities, can influence asymmetries in hand use (e.g., Hampson and Kimura, 1984). However, a consistent degree of right-hand preference has been reported for co-speech gestures, whether speakers are talking about verbal, spatial or neutral topics (Lavergne and Kimura, 1987), thus ruling out any effect of speech content on hand preference for gestures. In addition, in the 
present study, we can reasonably consider that the tasks were not complex enough to involve any problem-solving system that might interfere with handedness patterns. The manipulation task did not require any specific spatial ability, while in the pointing task, participants were simply asked to designate their favourite photograph, either simultaneously through speech and pointing gesture or solely through gesture. Moreover, the experimenter made sure that it was not difficult for the participants to briefly justify their choice in the speech condition (the latter were told that they did not have to provide any explanation if they did not know why they preferred a particular photograph).

Second, we cannot exclude the possibility that the participants, while inhibiting speech production in the silent condition, generated internal language when they chose and pointed towards a specific picture. Although the pattern of cerebral activation associated with internal speech would need to be investigated, this hypothesis might explain the equivalent degree of right-hand bias between pointing gestures produced on their own and gestures produced along with speech.

Finally, a simple explanation for the absence of any difference in the degree of asymmetry for pointing gestures between the speech and silent conditions would be that the right-sided asymmetry observed in human adults is already too strongly marked (much more so than in chimpanzees) for subtle differences in the intensity of activation of the relevant cerebral areas to increase it any further.

However, when we focused on the strength of hand preference (i.e., AbsHI), the right-sided asymmetry was found to be stronger for gestures produced in the silent condition than for gestures produced in the speech one. This result, while unexpected, does not necessarily invalidate the underlying hypothesis that a communication system in the left cerebral hemisphere controls both gestural and vocal communication, as hand-preference scores in the two conditions were still significantly correlated. However, this finding deserves further investigation. For example, participants may have produced co-speech gestures with their dominant hand in the speech condition. This would leave only their non-dominant hand free for pointing, leading to a stronger right-sided asymmetry in the silent condition. However, there are no data available so far demonstrating a greater bias for cospeech gestures than for pointing gestures in adults. Another future direction for research pertains to the different functions of gestural communication. When pointing gestures were produced on their own, they "shouldered" the full burden of communication, whereas they served more as props when they were produced along with speech, the latter playing the leading role. This difference has already been reported in adults: when participants are asked to communicate solely with their hands, their gestures take on the segmentation and combination properties characteristic of speech (Goldin-Meadow, 2006). In infants, a study using eventrelated potentials has highlighted developmental changes in the processing of gestures in the course of the second year (Sheehan et al., 2007). This study has revealed that common cerebral mechanisms initially underlie the mapping process for words and gestures, whereas subsequently, as children acquire language and no longer use gestures primarily as referential labels, words and gestures elicit distinct patterns of brain activity. Cerebral processes appear thus to be influenced by the role served by gestures, which, returning to the present study, further supports the hypothesis that the greater strength of hand preference for pointing in the silent condition is explained by the "language-like form" of gestures.

Moreover, results revealed significant correlations between hand-preference indexes for pointing gestures in the speech and silent conditions for right-handers (based on hand-preference scores for pointing in the silent condition), but not for left-handers or ambidextrous individuals. Lateralization of both pointing gestures and speech appears thus to be closely linked in right-handers for pointing, whereas patterns of functional asymmetries are less clearcut in other individuals, who are more likely to have either right-hemisphere language dominance or little lateralized specialisation.

Finally, handedness scores obtained from the questionnaire did not correlate with those obtained with the coordinated bimanual task. Further analyses revealed a significant, but moderate, correlation in right-handers only, reflecting less clearly lateralized patterns in left-handed and ambidextrous participants. Other studies have also reported weak correlations (explaining less than 25\% of the variance) between self-reported measures of handedness and more direct task-oriented measures (e.g., Bryden et al., 2000; Cavill and Bryden, 2003). It has been argued that these two methods may assess different aspects of hand preference, the hypothesis being that the questionnaire data reflect a more cognitive component (e.g., implying memory processes) and direct observation of hand use an immediate motor component (Cavill and Bryden, 2003).

The absence of a strong correlation between these two indexes emphasizes the importance of methodological choices in measuring hand-preference patterns. Therefore, researchers need to be aware of the differences between selfreported measures and direct observation of hand preference, as well as of the influence of the activity they select. For example, most of the items in the Edinburgh Inventory refer to unimanual activities (e.g., using a toothbrush or hitting a nail with a hammer), whereas the task administered in the present study required the coordination of both hands, which may also explain the different degrees of right-hand bias we recorded.

\section{Acknowledgements}

This research was supported by a French National Research Agency (ANR) grant, reference ANR-08-BLAN-0011_01.

\section{R E F E R E N C E S}

Annett M. Left, Right, Hand and Brain: The Right Shift Theory. Hillsdale, NJ: Erlbaum, 1985.

Barbieri F, Buonocore A, Dalla Volta R, and Gentilucci M. How symbolic gestures and words interact with each other. Brain and Language, 110: 1-11, 2009.

Bates E and Dick F. Language, gesture and the developing brain. Developmental Psychobiology, 40: 293-310, 2002. 
Bates E, O'Connell B, Vaid J, Sledge P, and Oakes L. Language and hand preference in early development. Developmental Neuropsychology, 2: 1-15, 1986.

Beaton AA. The nature and determinants of handedness. In Hugdahl $\mathrm{K}$ and Davidson RJ (Eds), The Asymmetrical Brain. Cambridge, MA: MIT Press, 2003: 105-158.

Bernardis P and Gentilucci M. Speech and gesture share the same communication system. Neuropsychologia, 44: 178-190, 2006.

Blake J, O'Rourke P, and Borzellino G. Form and function in the development of pointing and reaching gestures. Infant Behavior and Development, 17: 195-203, 1994.

Bonvillian JD, Richards HC, and Dooley TT. Early sign language acquisition and the development of hand preference in young children. Brain and Language, 58: 1-22, 1997.

Bourne VJ. Examining the relationship between degree of handedness and degree of cerebral lateralization for processing facial emotion. Neuropsychology, 22(3): 350-356, 2008.

Bryden MP, Free T, Gagné S, and Groff P. Handedness effects in the detection of dichotically-presented words and emotions. Cortex, 27: 229-235, 1991.

Bryden PJ, Pryde KM, and Roy EA. A performance measure of the degree of hand preference. Brain and Cognition, 44(3): 402-414, 2000.

Cavill S and Bryden PJ. Development of handedness: Comparison of questionnaire and performance-based measures of preference. Brain and Cognition, 53: 149-151, 2003.

$\mathrm{Chu} \mathrm{M}$ and Kita S. Co-speech gestures do not originate from speech production processes: Evidence from the relationship between co-thought and co-speech gestures. Proceedings of the 31st Annual Conference of the Cognitive Science Society, 591-595, 2009.

Cochet $\mathrm{H}$, Jover $\mathrm{M}$, and Vauclair J. Hand preference for pointing gestures and bimanual manipulation around the vocabulary spurt period. Journal of Experimental Child Psychology, in press.

Cochet $\mathrm{H}$ and Vauclair J. Pointing gestures produced by toddlers from 15 to 30 months: Different functions, hand shapes and laterality patterns. Infant Behavior and Development, 33: 432-442, 2010a.

Cochet $\mathrm{H}$ and Vauclair J. Features of spontaneous pointing gestures in toddlers. Gesture, 10(1): 86-107, 2010b.

Corina DP, San Jose-Robertson L, Guillemin A, High J, and Braun AR. Language lateralization in a bimanual language. Journal of Cognitive Neuroscience, 15: 718-730, 2003.

Dalby TJ, Gibson D, Grossi V, and Schneider RD. Lateralized hand gesture during speech. Journal of Motor Behavior, 12: 292-297, 1980.

Emmorey K, Mehta S, and Grabowski TJ. The neural correlates of sign versus word production. NeuroImage, 36: 202-208, 2007.

Everhart DE, Harrison DW, and Crews WD. Hemispheric asymmetry as a function of handedness: Perception of facial affect stimuli. Perceptual and Motor Skills, 82: 264-266, 1996.

Fagard J and Marks A. Unimanual and bimanual tasks and the assessment of handedness in toddlers. Developmental Science, 3(2): 137-147, 2000.

Fagard J and Lockman J. The effect of task constraints on infants' (bi)manual strategy for grasping and exploring objects. Infant Behavior and Development, 28: 305-315, 2005.

Fagot J and Vauclair J. Manual laterality in nonhuman primates: A distinction between handedness and manual specialization. Psychological Bulletin, 109: 76-89, 1991.

Flowers K. Handedness and controlled movement. British Journal of Psychology, 66: 39-52, 1975.

Gentilucci M and Dalla Volta R. Spoken language and arm gestures are controlled by the same motor control system. The Quarterly Journal of Experimental Psychology, 61: 944-957, 2008.

Goldin-Meadow S. Talking and thinking with our hands. Current Directions in Psychological Science, 15: 34-39, 2006.
Gómez JC. Requesting gestures in captive monkeys and apes. Conditioned responses or referential behaviours? Gesture, 5: 89-103, 2005.

Gonzalez CLR and Goodale MA. Hand preference for precision grasping predicts language lateralization. Neuropsychologia, 47(14): 3182-3189, 2009.

Grossi G, Semenza C, Corazza S, and Volterra V. Hemispheric specialization for sign language. Neuropsychologia, 34: 737-740, 1996.

Hampson E and Kimura D. Hand movement asymmetries during verbal and nonverbal tasks. Canadian Journal of Psychology, 38: 102-125, 1984.

Healey JM, Liederman J, and Geschwind N. Handedness is not a unidimensional trait. Cortex, 22: 33-53, 1986.

Hopkins WD. On the other hand: Statistical issues in the assessment and interpretation of hand preference data in nonhuman primates. International Journal of Primatology, 20: 851-866, 1999.

Hopkins WD and Cantero M. From hand to mouth in the evolution of language: The influence of vocal behaviour on lateralized hand use in manual gestures by chimpanzees. Developmental Science, 6: 55-61, 2003.

Hopkins WD, Russell J, Freeman H, Buehler N, Reynolds E, and Schapiro SJ. The distribution and development of handedness for manual gestures in captive chimpanzees (Pan troglodytes). Psychological Science, 16: 487-493, 2005.

Iverson JM and Thelen E. Hand, mouth and brain: The dynamic emergence of speech and gesture. Journal of Consciousness Studies, 6: 19-40, 1999.

Kimura D. Manual activity during speaking: I. Right-handers. Neuropsychologia, 11: 45-50, 1973.

Knecht S, Dräger B, Deppe M, Bobe L, Lohmann H, Flöel A, et al. Handedness and hemispheric language dominance in healthy humans. Brain, 123: 2512-2518, 2000.

Kraus EH. Handedness in children. In Henderson A and Pehoski C (Eds), Hand Function in the Child: Foundations for Remediation. St. Louis, MO: Mosby, 2005: 161-191.

Lang PJ, Bradley MM, and Cuthbert BN. The International Affective Pictures System (IAPS). Technical Manual and Affective Ratings. Gainesville, FL: University of Florida, 1999.

Lausberg $\mathrm{H}$ and Kita $\mathrm{S}$. The content of the message influences the hand preference in co-speech gestures and in gesturing without speaking. Brain and Language, 86: 57-69, 2003.

Lavergne $J$ and Kimura D. Hand movement asymmetry during speech: No effect of speaking topic. Neuropsychologia, 25: 689-693, 1987.

McManus IC, Sik G, Cole DR, Mellon AF, Wong J, and Kloss J. The development of handedness in children. British Journal of Developmental Psychology, 6: 257-273, 1988.

Medland SE, Perelle I, De Monte V, and Ehrman L. Effects of culture, sex, and age on the distribution of handedness: An evaluation of the sensitivity of three measures of handedness. Laterality, 9(3): 287-297, 2004.

Meguerditchian A and Vauclair J. Contrast of hand preferences between communicative gestures and non communicative actions in baboons: Implications for the origins of hemispheric specialization for language. Brain and Language, 108: 167-174, 2009.

Meguerditchian A, Vauclair J, and Hopkins WD. Captive chimpanzees use their right hand to communicate with each other: Implications for the origin of the cerebral substrate for language. Cortex, 46: 40-48, 2010.

Montgomery KJ, Isenberg N, and Haxby JV. Communicative hand gestures and object-directed hand movements activated the mirror neuron system. Social Cognitive and Affective Neuroscience, 2: 114-122, 2007.

Oldfield RC. The assessment and analysis of handedness: The Edinburgh inventory. Neuropsychologia, 9: 97-113, 1971. 
Özyürek A, Willems RM, Kita S, and Hagoort P. On-line integration of semantic information from speech and gesture: Insights from event-related brain potentials. Journal of Cognitive Neuroscience, 19: 605-616, 2007.

Pierno AC, Tubaldi F, Turella L, Grossi P, Barachino L, Gallo P, et al. Neurofunctional modulation of brain regions by the observation of pointing and grasping actions. Cerebral Cortex, 19(2): 367-374, 2009.

Pika S. Gestures of apes and pre-linguistic human children: Similar or different? First Language, 28: 116-140, 2008.

Provins KA and Glencross DJ. Handwriting, typewriting and handedness. Quarterly Journal of Experimental Psychology, 10: 282-289, 1968.

Raymond $\mathrm{M}$ and Pontier D. Is there geographical variation in human handedness? Laterality, 9: 35-52, 2004.

Saucier DM and Elias LJ. Lateral and sex differences in manual gesture during conversation. Laterality, 6: 239-245, 2001.
Sheehan EA, Namy LL, and Mills DL. Developmental changes in neural activity to familiar words and gestures. Brain and Language, 101: 246-259, 2007.

Steiger JH. Tests for comparing elements of a correlation matrix. Psychological Bulletin, 87: 245-251, 1980.

Vaid J, Bellugi U, and Poizner H. Hand dominance for signing: Clues to brain lateralization. Neuropsychologia, 27: 949-960, 1989.

Vauclair J and ImbaultJ. Relationships between manual preferences for manipulative actions and for pointing gestures in infants and toddlers. Developmental Science, 12: 1060-1069, 2009.

Xu J, Gannon PJ, Emmorey K, Smith JF, and Braun AR. Symbolic gestures and spoken language are processed by a common neural system. Proceedings of the National Academy of Sciences, 106(49): 20664-20669, 2009.

Young AM, Lock AJ, and Service V. Infants' hand preferences for actions and gestures. Developmental Neuropsychology, 1: $17-27,1985$ 